\title{
Lifted system iterative learning control applied to an industrial robot
}

\author{
W.B.J. Hakvoort \\ Netherlands Institute for Metals Research \\ University of Twente \\ P.O. Box 217, 7500 AE Enschede \\ w.hakvoort@nimr.nl
}

\section{Introduction}

Laser welding requires accurate manipulation of the laser beam over the materials to be joined to ensure defect free welds. From an industrial perspective the use of multi-joint industrial robots for laser welding is attractive as they can track complicated seam geometries and are relatively inexpensive. However, for some seam-geometries the accuracy of these robots is insufficient for laser welding. The goal of this research is to improve the tracking accuracy of the Stäubli RX90 robot (Figure 1) by adding an iterative learning controller to the standard industrial controller.

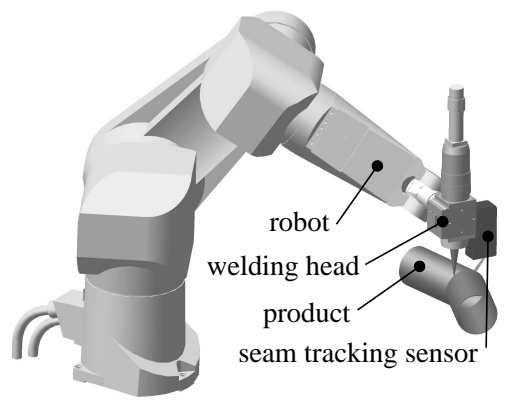

Figure 1: Stäubli RX90 robot

\section{Lifted system ILC}

Iterative learning control improves the tracking accuracy of systems that make repetitive movements by updating a feedforward using measurements of the tracking error in previous trials. In this research the lifted system description has been used to design an iterative learning controller for an industrial robot. The lifted system description was previously exploited for iterative learning control of a linear time invariant system by Dijkstra [1].

In the lifted system description all time samples of a (multidimensional) signal are put in a single vector, the lifted vector. A linear time-variant system is described by a large constant matrix that maps a lifted input vector to a lifted output vector. A linear time-variant model of the Stäubli RX90 robot has been obtained by linearisation of a non-linear dynamic model for small perturbations around the reference trajectory. The non-linear dynamic robot model was developed by Waiboer [2].

\author{
R.G.K.M. Aarts, J. van Dijk and J.B. Jonker \\ University of Twente \\ Laboratory of Mechanical Automation \\ P.O. Box 217, 7500 AE Enschede \\ \{r.g.k.m.aarts, j.vandijk, j.b.jonker\}@utwente.nl
}

Using the lifted system description an iterative learning controller can be considered as a digital feedback controller acting on a high-dimensional system. An effective iterative learning controller for the Stäubli RX90 robot has been designed by the application of the internal model principle

\section{Results}

The lifted system ILC was tested with the joint motions required to let the robot-tip track two perpendicular horizontal lines interconnected by an arc with a radius of $100 \mathrm{~mm}$. The trajectory was tracked with $350 \mathrm{~mm} / \mathrm{s}$. The tracking error of the joints reduced considerably in a few iterations as shown in figure 2. The lifted approach thus yields a fast converging iterative learning controller for an industrial robot that can be applied in addition to the conventional industrial feedback controller.

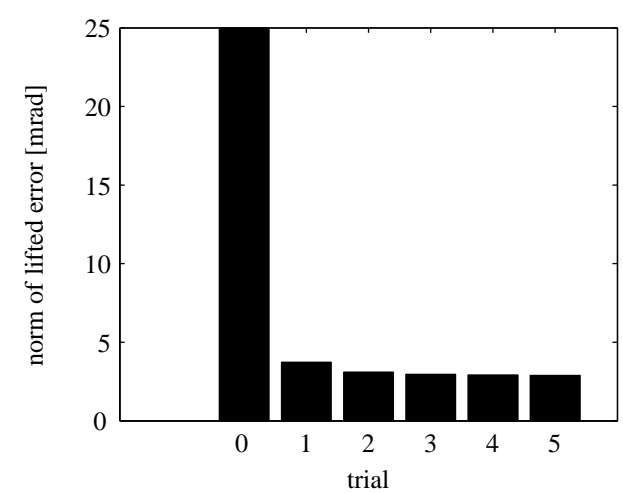

Figure 2: Norm of lifted error for trial 0 (no learning) to trial 5.

\section{Future work}

The lifted system ILC will be extended to control the tip motion of the robot using measurements of a seam tracking sensor (see figure 1).

\section{References}

[1] Dijkstra B.G., Iterative Learning Control with applications to a wafer-stage, $\mathrm{PhD}$ Thesis, T.U. Delft, The Netherlands, September 2004

[2] Waiboer R.R., Dynamic Robot Modelling, Identification and Simulation, PhD Thesis, University of Twente, The Netherlands, to be published 2005 